\title{
HIGHLIGHTS
}

\section{Coated-platelets can predict risk of Alzheimer disease}

An assay for elevated coated-platelet levels can be used to predict which patients with mild cognitive impairment (MCI) are most likely to progress rapidly to Alzheimer disease (AD), according to a new study. "Coated-platelets represent a peripheral biomarker of progression risk of AD," explains lead investigator Calin Prodan, "and few such biomarkers are available."

Levels of coated-platelets-a subclass of platelets generated on dual-agonist stimulation by collagen and thrombinvary in the general population, but are high in the early stages of $\mathrm{AD}$. The reason for this finding is as yet unknown, although Prodan suggests a connection to the etiology of AD through either altered amyloid metabolism or AD-associated inflammatory processes.

Initial coated-platelet levels were measured in 74 patients with amnestic $\mathrm{MCI}$, the conditions of whom were monitored for the next 3 years, with a median follow-up of 24 months. The patients were categorized into tertiles at the start of the study, on the basis of their coated-platelet levels (lower tertile: 7.0-26.0\%, middle tertile: $26.1-40.0 \%$, and upper tertile: $40.1-60.0 \%)$.

Prodan and colleagues observed that $4 \%$, $13 \%$ and $37 \%$ of individuals in the study developed $\mathrm{AD}$ in the lower, middle and upper tertiles, respectively. The hazard rate for progression of patients to $\mathrm{AD}$ in the upper tertile was 5.1 times that of patients in the lowest tertile, but little difference was seen in the hazard rates for those in the middle and lowest tertile groups.

The team are working on a way to simplify the assay, which is currently impractical in routine clinical analysis as it involves flow cytometry of platelets activated with thrombin and convulxin-a protein obtained from rattlesnake venom. Until this assay is simplified, "the largescale prospective studies required for determining whether elevated coatedplatelet levels are observable in individuals

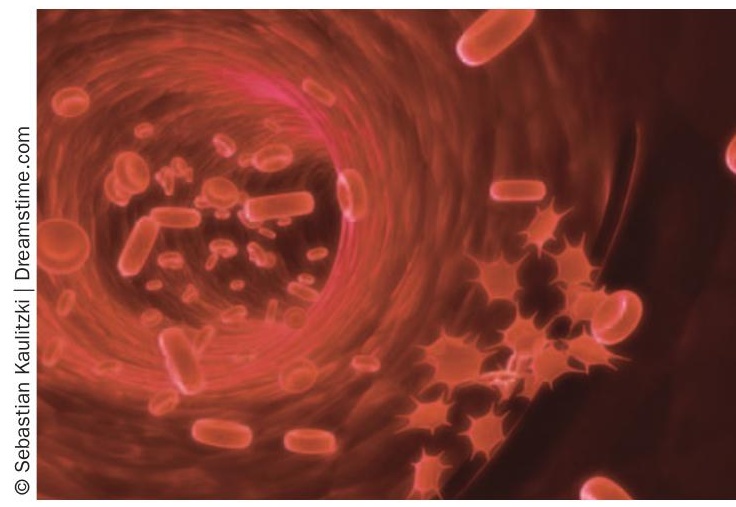

before the chronological onset of MCI are technically unfeasible," says Prodan.

Highlighting the importance of continuing this research, Prodan concludes "identification of patients most vulnerable to rapid progression to dementia would greatly facilitate pharmacological trials of medications designed to prevent AD."

Carolyn McSharry

Original article Prodan, C. I. et al. Coated-platelet levels and progression from mild cognitive impairment to Alzheimer disease. Neurology 76, 247-252 (2011) 